\title{
Etude de cohérence entre aspect relationnel et satisfaction des apprenants à travers la pédagogie différenciée
}

\section{Coherence study between relational aspect and learner satisfaction through the differentiated pedagogy}

\author{
Okacha Diyer ${ }^{1 *}$, Naceur Achtaich ${ }^{1}$, and Khalid Najib ${ }^{2}$ \\ ${ }^{1}$ Faculté des Sciences Ben M'Sik, Université Hassan II de Casablanca, Maroc \\ ${ }^{2}$ Ecole Nationale Supérieure des Mines de Rabat, Maroc
}

\begin{abstract}
Résumé. La pédagogie différenciée offre aux élèves l'opportunité de développer des interactions locales au sein de leurs groupes et aussi au sein $\mathrm{du}$ groupe-classe. Afin de réaliser les objectifs pour lesquels l'enseignant adopte une méthode qui se base sur cette pédagogie, il devra préparer les conditions préalables de sa réussite. Dans notre recherche, nous nous sommes intéressés à l'aspect relationnel qui peux exister entre les membres de chaque groupe afin de créer un environnement motivant et ambitieux. Pour mettre en évidence l'importance du critère relationnel pour une partition donnée d'une classe, nous avons modélisé cet aspect par une fonction appelée fonction relationnelle globale sur toute la classe dont sa restriction sur tout groupe est appelée fonction relationnelle locale. Pour prouver l'impact de l'aspect relationnel sur l'efficacité de l'opération d'apprentissage, nous avons aussi mis en évidence une autre fonction qui caractérise la satisfaction des élèves de la classe, appelée fonction de satisfaction globale sur toute la classe dont sa restriction sur tout groupe est appelée fonction de satisfaction locale. Nous avons étudié quelques propriétés de ces deux fonctions et ensuite nous avons mis en évidence la corrélation qui existe entre le relationnel et le rendement des apprenants dans le groupe-classe.
\end{abstract}

\begin{abstract}
The differentiated pedagogy offers students the opportunity to develop local interactions within their groups and also within the class group. In order to achieve the objectives for which the teacher adopts a method based on this pedagogy, he must prepare the prerequisites for his success. In our research, we were interested in the relational aspect that can exist between the members of each group in order to create a motivating and an ambitious environment. To highlight the importance of the relational criterion for a given partition of a class, we have modeled this aspect by a function called global relational function on the whole class
\end{abstract}

* Corresponding author: odiyer@yahoo.fr 
whose restriction on any group is called local relational function. To prove the impact of the relational aspect on the effectiveness of the learning operation, we have also highlighted another function that characterizes the students' satisfaction in the class, called overall satisfaction function over the whole class whose restriction on any group is called local satisfaction function. We have studied some properties of these two functions and then we have highlighted the correlation that exists between the relational and the performance of learners in the group-class.

\section{Introduction}

Plusieurs chercheurs se sont intéressés à la pédagogie différenciée et à son impact sur l'opportunité qu'elle procure aux apprenants pour différentes acquisitions des savoirs, savoir-faire et savoir-être. Les conditions préalables chez les apprenants ne sont pas les mêmes tandis que les objectifs finaux de l'opération de l'apprentissage le sont, ce qui donne l'importance à introduire la pédagogie différenciée dans notre enseignement. Dans ce cadre nous citons les sept postulats de Burn [1], Qui conjoncture le fait que :

- Il n'y a pas deux apprenants qui progressent à la même vitesse.

- Il n'y a pas deux apprenants qui soient prêts à apprendre en même temps.

- Il n'y a pas deux apprenants qui utilisent les mêmes techniques d'étude.

- Il n'y a pas deux apprenants qui résolvent les problèmes exactement de la même manière.

- Il n'y a pas deux apprenants qui possèdent le même répertoire de comportements.

- Il n'y a pas deux apprenants qui possèdent le même profil d'intérêt.

- Il n'y a pas deux apprenants qui soient motivés pour atteindre les mêmes objectifs.

En fait, l'enseignant en général, veut transmettre avec efficacité des compétences qui sont composées de savoirs, savoir-faire et savoir-être à tous les apprenants. L'opération est réussite lorsque l'évaluation finale donne une satisfaction à tous les acteurs pédagogiques concernés. Ainsi, le fait de s'intéresser à cette pédagogie est important dans le cadre de préparer des conditions d'équités pour tous les élèves. De plus les interactions au sein de chaque groupe sont aussi intéressantes de sorte que les élèves se communiquent avec efficacité entre eux. Touchard s'est intéressé aux conditions préalables pour appliquer une méthode basée sur la pédagogie différenciée [2]. En effet, les enseignants doivent faire une évaluation diagnostique avant d'entamer une répartition des élèves à savoir leurs prérequis cognitifs. Dans son travail il a présenté les moyens et les procédures à préparer par l'enseignant avant, pendant et après l'application d'une méthode qui se repose sur la pédagogie différenciée. Herreman et al. ont montré que les stratégies appliquées par l'enseignant se basent sur une évaluation précise en commençant par un diagnostic de l'état initial des apprenants [3]. Il doit prendre en considération les caractéristiques cognitives des apprenants qui peuvent s'évoluer avec rentabilité, suivant des objectifs en tenant compte des interactions productives. Volpe a présenté quelques dispositifs de différentiation qui se composent du plan de travail, les groupes de besoins, les ateliers d'apprentissage et le tutorat entre élèves [4]. Touchard a cité parmi les variables importantes pour différencier les élèves est l'organisation de la classe comme étant un axe important dans la pédagogie différenciée [5]. Dans laquelle il a donné les objectifs des interactions entre les membres d'un groupe. Il assume que dans le cas général, suivant des critères cognitifs, la classe est soit homogène ou soit hétérogène. Nous citons aussi la recherche de Roux qui a mis la théorie du socioconstructivisme au cœur de toute opération d'apprentissage [6]. En effet, l'acquisition du Savoir fait intervenir non seulement les mécanismes cognitifs personnels mais aussi l'environnement social qui fait engager d'autres acteurs. Nous citons aussi la théorie de Vygotsky [7] qui a relié le développement cognitif avec les interactions qui peuvent se produire dans l'environnement social des apprenants, ce qui met en valeur, d'une 
façon importante, le contexte psychologique. Nous évoquons la recherche de Clerc [8] qui a montré que l'élève lors de ses interactions dans la classe vit une sorte de conflit intrinsèque de présenter sa difficulté d'apprentissage devant les autres intervenants de peur d'être sousestimé. Elle a mis le point sur la fait que si un apprenant n'a pas une position relationnelle forte au sein du groupe, il a la tendance de cacher sa difficulté et de se fondre dans la masse. En fait c'est un problème psychologique qui est posé par l'individu lui-même, en faisant des mauvaises projections sur ses interventions éventuelles. Connac a confirmé que l'environnement social de l'apprenant est riche en interactions pédagogiques et qui influence sur la motivation de l'apprenant à s'impliquer davantage dans son apprentissage [9]. Nous citons la recherche de Tyng CM et al. qui ont montré l'importance de l'émotion dans les interactions, ces dernières créent une motivation intrinsèque qui propulsent des organes du cerveau à se manifester pour atteindre une performance de créativité [10]. Saul McLeod s'est intéressé à la zone de développement proximal, où il a montré que les conseils et les encouragements de l'entourage de l'apprenant peut influencer sur la réalisation d'un enfant d'une façon importante mieux que s'il est laissé tout seul a affronté une situation d'apprentissage [11]. Plusieurs chercheurs se sont intéressés aux relations qui existent entre l'enseignant et ses élèves et son impact sur la réussite de l'opération d'apprentissage dans les écoles maternelles, primaires et secondaires $[12,13]$. Gerda Hagenauer et Simone E. Volet, ont montré l'importance de l'étude de la qualité de la relation enseignant-étudiant à l'université sur la nature de l'enseignement à ce niveau [14]. Nous citons aussi, le travail de Croxton qui s'est intéressé au rôle de l'interactivité dans la satisfaction et la persévérance des étudiants dans l'apprentissage en ligne [15]. En effet il a proposé un cadre d'interactivité de cours en ligne qui est basé sur des critères d'environnements pour produire la satisfaction des apprenants et ensuite leur persistance. Dans notre travail, nous évoquons l'importance de choix des groupes par les élèves eux-mêmes sur l'environnement de satisfaction qui peut exister au sein de chaque groupe. Ainsi, nous mettons en évidence l'impact de l'aspect relationnel entre les membres d'un groupe sur la motivation des élèves, ainsi sur leurs rendements pédagogiques. L'article est organisé de la façon suivante, dans la section 2, nous présentons la problématique et la méthodologie de notre recherche. Nous fournissons une étude théorique des deux aspects relationnel et satisfaction de l'opération d'apprentissage en les modélisant sous formes de deux fonctions. Ensuite, nous présentons une étude de l'impact du relationnel sur la satisfaction des apprenants. Dans la section 3, nous offrons les résultats et les discussions, où nous présentons une application de l'approche utilisée et des interprétations des résultats obtenus. Nous terminons, dans la section 4, par une conclusion.

\section{Problématique et Méthodologie}

Nous présentons tout d'abord la problématique de notre recherche et ensuite, nous fournissons la méthodologie scientifique pour répondre à cette problématique.

\subsection{Problématique}

Le souci principal des chercheurs qui s'intéressent à la pédagogie différenciée est de trouver les critères prépondérants pour une partition en groupes d'une classe, afin de préparer un environnement pédagogique motivant. Nous ne devrons pas seulement s'intéresser aux prérequis cognitifs des élèves pour former des partitions d'une classe mais aussi prendre en considération les interactions qui peuvent se produire dans cette partition.

Dans le cadre de préparer les conditions pédagogiques favorables, dans la pédagogie différenciée, pour un apprentissage motivant et judicieux, l'enseignant doit s'intéresser aux critères de composition des groupes. Parmi les facteurs prépondérants pour réaliser cet 
objectif, il existe l'environnement de l'apprenant. Dans cette perspective, quelle corrélation peut-elle exister entre l'aspect relationnel régnant au sein des groupes et le rendement de l'opération d'apprentissage?.

\subsection{Méthodologie}

Pour trouver une corrélation entre l'aspect relationnel existant dans chaque groupe d'une partition en adoptant la pédagogie différenciée et la motivation des apprenants, nous avons modélisé ces deux caractères. Le premier par une fonction appelée relationnelle locale dans un groupe et le deuxième est caractérisé par une autre fonction appelée satisfaction locale dans un groupe. La fonction relationnelle locale sur un groupe prend comme argument un membre de ce groupe et son image est en relation avec la résultante des amplitudes des degrés de relation de celui-ci avec les autres membres de son groupe en adoptant l'échelle de Likert à cinq modalités. Nous avons relié la fonction de satisfaction locale avec l'autoévaluation de la progression d'acquérir l'information de chaque membre du groupe. Ainsi, nous avons procédé à une extension des deux fonctions précédentes sur la classe toute entière afin que nous pouvons faire une étude globale. Nous avons appliqué l'approche précédente à un échantillon d'une classe scientifique dans le cycle secondaire qualifiant de 30 élèves pour une séance de mathématiques.

L'aspect relationnel entre les membres de chaque groupe, permet l'existence d'une satisfaction intéressante au sein de chacun. Ce qui donne à chaque élément l'opportunité de poser sa difficulté aux autres membres sans peur de la sous-estime. Le fait que les groupes sont formés à partir de choix des élèves pour constituer leurs groupes, donne la motivation nécessaire à travailler ensemble et à aider son ami-camarade. Nous voulons, dans ce paragraphe, modéliser cet aspect relationnel par des formules mathématiques. Pour cela, nous nous intéressons à ce qui se passe localement dans un groupe. Soit $\mathrm{G}$ un groupe de la répartition, tel que le cardinal de $G$ est égal à $r$, posons $G=\left\{m_{1}, m_{2}, \ldots m_{r}\right\}$.

\subsubsection{Fonction relationnelle}

Nous considérons l'application suivante :

$$
\varphi_{/ G}: G \rightarrow \mathbb{N} \text { telle que } m_{i} \rightarrow \varphi_{/ G}\left(m_{i}\right)=n_{i}
$$

où $n_{i}=\sum_{\substack{j=1 \\ j \neq i}}^{r} r_{i j}, r_{i j}$ est le coefficient qui est déterminé par la quantification du degré d'amitié du membre $m_{i}$ avec le membre $m_{j}$, en adoptant l'échelle de Likert suivant, voir Tableau 1.

Tableau 1. Quantification de l'appréciation de la relationnelle des élèves suivant l'échelle de Likert.

\begin{tabular}{|c|c|}
\hline Relation & Quantification du degré de l'amitié \\
\hline Excellente & 5 \\
\hline Bonne & 4 \\
\hline Moyenne & 3 \\
\hline Faible & 2 \\
\hline Très faible & 1 \\
\hline
\end{tabular}

Nous appelons $\varphi_{/ G}$ la fonction relationnelle locale définie sur $G$. Nous présentons quelques propriétés de la fonction $\varphi_{/ G}$, 


$$
\begin{aligned}
& \text { pour tout } 1 \leq i \leq r, \quad r-1 \leq \varphi_{/ G}\left(m_{i}\right) \leq 5(r-1) \\
& r(r-1) \leq \sum_{i=1}^{r} \varphi_{/ G}\left(m_{i}\right) \leq 5 r(r-1) .
\end{aligned}
$$

Nous introduisons, ensuite, une fonction globale sur toute la classe notée $\varphi$ définie de la façon suivante : soit $G_{1}, G_{2}, \ldots, G_{q}$ une partition de la classe $C,\left(C=G_{1} \cup G_{2} \cup \ldots \cup G_{q}\right)$, pour tout élève $E$ de la classe $C$, il existe i entre 1 et $r$, il existe un unique $j$ entre 1 et $q$ tels que $E=m_{i, j}$ est dans $G_{j}$,

$$
\varphi(E)=\varphi\left(m_{i, j}\right)=\varphi_{\mid G j}\left(m_{i, j}\right)
$$

Nous appelons $\varphi$ la fonction relationnelle globale définie sur toute la classe C, ainsi,

$$
\varphi(C)=\coprod_{j=1}^{q} \varphi_{\mid G j}\left(G_{j}\right)
$$

Notons pour tout entier $\mathrm{j}$ compris entre 1 et $\mathrm{q}$,

$$
\begin{aligned}
& G_{j}=\left(m_{1, j}, m_{2, j}, \ldots, m_{r, j}\right), \\
& \varphi\left(G_{j}\right)=\left(\varphi\left(m_{1, j}\right), \varphi\left(m_{2, j}\right), \ldots, \varphi\left(m_{r, j}\right)\right), \\
& M_{j}=\operatorname{moyenne}\left(\varphi\left(G_{j}\right)\right)=\frac{1}{r} \sum_{i=1}^{r} \varphi\left(m_{i, j}\right)=\frac{1}{r} \sum_{i=1}^{r} \varphi_{\mid G j}\left(m_{i, j}\right)
\end{aligned}
$$

Nous pouvons conclure à partir de $M_{j}$, voir Tableau 2, l'environnement d'amitié qui règne au sein du groupe $G_{j}$.

Tableau 2. Appréciation de l'environnement relationnel au sein d'un groupe.

\begin{tabular}{|c|c|}
\hline Valeur de $\boldsymbol{M}_{\boldsymbol{j}}$ & Appréciation de l'environnement d'amitié dans le groupe $\boldsymbol{G}_{\boldsymbol{j}}$ \\
\hline $4(r-1)<M_{j} \leq 5(r-1)$ & Très bonne \\
\hline$\frac{10}{3}(r-1)<M_{j} \leq 4(r-1)$ & Bonne \\
\hline$\frac{8}{3}(r-1)<M_{j} \leq \frac{10}{3}(r-1)$ & Assez bonne \\
\hline $2(r-1)<M_{j} \leq \frac{8}{3}(r-1)$ & Juste moyenne \\
\hline$r-1 \leq M_{j} \leq 2(r-1)$ & Faible \\
\hline
\end{tabular}

Et par conséquent, l'environnement d'amitié qui règne au sein de la classe est déterminé à partir des moyens de la relationnelle des groupes d'une classe. Ainsi la valeur $M=\frac{1}{q} \sum_{j=1}^{q} M_{j}$, nous donne une information sur le niveau relationnel qui existe au sein de la totalité des groupes de la classe. Le Tableau 3, nous indique le niveau du climat relationnel qui existe dans la classe, en adoptant une méthode qui base sur la pédagogie différenciée. 
Tableau 3. Appréciation de l'environnement relationnel au sein de la classe.

\begin{tabular}{|c|c|}
\hline Valeur de $\boldsymbol{M}$ & $\begin{array}{c}\text { Appréciation sur le climat relationnel dans la } \\
\text { classe }\end{array}$ \\
\hline $4(r-1)<M \leq 5(r-1)$ & Très bonne \\
\hline$\frac{10}{3}(r-1)<M \leq 4(r-1)$ & Bonne \\
\hline$\frac{8}{3}(r-1)<M \leq \frac{10}{3}(r-1)$ & Assez bonne \\
\hline $2(r-1)<M \leq \frac{8}{3}(r-1)$ & Juste moyenne \\
\hline$r-1 \leq M \leq 2(r-1)$ & Faible \\
\hline
\end{tabular}

Nous étudions la fonction $\varphi_{/ \mathrm{G}}$ pour déterminer l'amplitude local de l'aspect relationnel dans un seul groupe, et nous étudions la fonction $\varphi$ pour déterminer l'amplitude global de l'aspect relationnel dans une classe.

\subsubsection{Fonction de satisfaction}

Tout d'abord, nous définissons le sens de la satisfaction d'un membre du groupe, nous pouvons la caractériser par les champs suivants : la facilité de poser les questions sans crainte d'être sous-estimé, la bonne progression dans l'acquisition de l'information, le respect de chaque membre du groupe et la volonté de continuer la discussion sur le contenu après la séance.

Maintenant, nous considérons l'application suivante,

$$
\theta_{/ G}: G \rightarrow \mathbb{N} \text { telle que } m_{i} \rightarrow \theta_{/ G}\left(m_{i}\right)=s_{i}
$$

où, $s_{i}$ est le coefficient qui est déterminé par la quantification du degré de satisfaction du membre $m_{i}$ dans son groupe, en adoptant l'échelle de Likert, voir Tableau 4.

Tableau 4. Quantification de l'appréciation de la satisfaction des élèves suivant l'échelle de Likert.

\begin{tabular}{|c|c|}
\hline Satisfaction & Quantification du degré de satisfaction \\
\hline Excellente & 5 \\
\hline Bonne & 4 \\
\hline Moyenne & 3 \\
\hline Faible & 2 \\
\hline Très faible & 1 \\
\hline
\end{tabular}

Nous appelons $\theta_{/ G}$ la fonction de satisfaction locale définie sur G. Nous présentons des propriétés de la fonction $\theta_{/ G}$,

$$
\begin{aligned}
& \text { pour tout } 1 \leq i \leq r, 1 \leq \theta_{/ G}\left(m_{i}\right) \leq 5, \\
& r \leq \sum_{i=1}^{r} \theta_{/ G}\left(m_{i}\right) \leq 5 r
\end{aligned}
$$

Nous introduisons une fonction globale sur toute la classe notée $\theta$ définie de la façon suivante:

soit $G_{1}, G_{2}, \ldots, G_{q}$ une partition de la classe $C,\left(C=G_{1} \cup G_{2} \cup \ldots \cup G_{q}\right)$, pour tout élève $E$ de la classe $C$, il existe i entre 1 et $r$, il existe un unique j entre 1 et $q$ tels que $E=m_{i, j}$ est dans $G_{j}$, 


$$
\theta(E)=\theta\left(m_{i, j}\right)=\theta_{\mid G j}\left(m_{i, j}\right)
$$

Nous appelons $\theta$ la fonction de satisfaction globale définie sur toute la classe $C$, ainsi,

$$
\theta(C)=\coprod_{j=1}^{q} \theta_{\mid G j}\left(G_{j}\right)
$$

Nous étudions la fonction $\theta_{/ G}$ pour déterminer l'amplitude local de satisfaction et par suite sur le rendement de l'opération d'apprentissage dans un seul groupe. Nous étudions la fonction $\theta$ pour étudier l'amplitude de la satisfaction globale et par suite sur le rendement de l'opération d'apprentissage dans la classe toute entière.

Notons pour tout entier $j$ compris entre 1 et $q$,

$$
\begin{aligned}
& G_{j}=\left(m_{1, j}, m_{2, j}, \ldots, m_{r, j}\right), \\
& \theta\left(G_{j}\right)=\left(\theta\left(m_{1, j}\right), \theta\left(m_{2, j}\right), \ldots, \theta\left(m_{r, j}\right)\right), \\
& N_{j}=\operatorname{moyenne}\left(\theta\left(G_{j}\right)\right)=\frac{1}{r} \sum_{i=1}^{r} \theta\left(m_{i, j}\right) .
\end{aligned}
$$

Nous pouvons, suivant le Tableau 5, conclure à partir de $N_{j}$ l'environnement de satisfaction qui règne au sein du groupe $G_{j}$.

Tableau 5. Appréciation de l'environnement de satisfaction au sein d'un groupe.

\begin{tabular}{|c|c|}
\hline Valeur de $\boldsymbol{N}_{\boldsymbol{j}}$ & Appréciation de l'environnement de satisfaction dans le groupe $\boldsymbol{G}_{\boldsymbol{j}}$ \\
\hline $4<N_{j} \leq 5$ & Très bonne \\
\hline$\frac{10}{3}<N_{j} \leq 4$ & Bonne \\
\hline$\frac{8}{3}<N_{j} \leq \frac{10}{3}$ & Assez bonne \\
\hline $2<N_{j} \leq \frac{8}{3}$ & Juste moyenne \\
\hline $1 \leq N_{j} \leq 2$ & Faible \\
\hline
\end{tabular}

L'environnement de satisfaction qui règne au sein de la classe est calculé à partir des moyens de satisfactions des groupes d'une classe. Ainsi $R=\frac{1}{q} \sum_{j=1}^{q} N_{j}$, nous donne une information sur le niveau de satisfaction qui existe au sein de la classe. Ainsi le Tableau 6 nous indique, en adoptant une méthode qui se base sur la pédagogie différenciée, la nature du climat de satisfaction qui règne dans la classe.

Tableau 6. Appréciation de l'environnement de satisfaction au sein de la classe.

\begin{tabular}{|c|c|}
\hline Valeur de $R$ & Appréciation sur le climat de satisfaction dans la classe \\
\hline $4<R \leq 5$ & Très bonne \\
\hline$\frac{10}{3}<R \leq 4$ & Bonne \\
\hline$\frac{8}{3}<R \leq \frac{10}{3}$ & Assez bonne \\
\hline $2<R \leq \frac{8}{3}$ & Juste moyenne \\
\hline $1 \leq R \leq 2$ & Faible \\
\hline
\end{tabular}




\subsubsection{Etude de l'impact du relationnel sur la satisfaction des apprenants}

$\mathrm{Au}$ sein de chaque groupe, nous mettons en évidence l'impact de l'environnement relationnel sur la satisfaction des membres de ce groupe. $\grave{A}$ partir des composantes correspondantes des deux vecteurs $\varphi\left(G_{j}\right)$ et $\theta\left(G_{j}\right)$, nous déterminons en valeur, voir Tableau 7, la corrélation entre le relationnel et le rendement des apprenants dans leur groupe.

Tableau 7. Corrélation entre le relationnel et la satisfaction de chaque membre.

\begin{tabular}{|c|c|}
\hline $\boldsymbol{\varphi}\left(\boldsymbol{G}_{\boldsymbol{j}}\right)$ & $\boldsymbol{\theta}\left(\boldsymbol{G}_{\boldsymbol{j}}\right)$ \\
\hline$\varphi\left(m_{1, j}\right)$ & $\theta\left(m_{1, j}\right)$ \\
\hline$\varphi\left(m_{2, j}\right)$ & $\theta\left(m_{2, j}\right)$ \\
\hline$\ldots$ & $\ldots$ \\
\hline$\ldots$ & $\ldots$ \\
\hline$\varphi\left(m_{r, j}\right)$ & $\theta\left(m_{r, j}\right)$ \\
\hline
\end{tabular}

Pour mettre en évidence l'importance de la relationnelle sur le rendement de tous les membres de la classe, nous pouvons déterminer la corrélation entre les deux vecteurs suivants,

$$
\boldsymbol{M} \boldsymbol{M}=\left(M_{1}, M_{2}, \ldots, M_{q}\right) \text { et } \boldsymbol{R} \boldsymbol{R}=\left(N_{1}, N_{2}, \ldots, N_{q}\right)
$$

\section{Résultats et discussion}

Nous mettons en application la recherche précédente en considérant un échantillonnage formé par une classe de 30 élèves et qui sont répartis en groupes de 5 élèves chacun. Soit $G_{1}, G_{2}, G_{3}, G_{4}, G_{5}$ et $G_{6}$ une partition de la classe $C,\left(C=G_{1} \cup G_{2} \cup G_{3} \cup G_{4} \cup G_{5} \cup\right.$ $G_{6}$ ), pour tout élève $E$ de la classe $C$, il existe i entre 1 et 5 , il existe un unique $j$ entre 1 et 6 tels que $E=m_{i, j}$ est dans $G_{j}$.

\subsection{Application de l'approche utilisée}

Nous présentons les deux fonctions relationnelles et de satisfaction qui sont définies auparavant dans ce cas pratique dont l'objectif est de déterminer la cohérence qui existe entre l'aspect relationnel et la satisfaction des apprenants.

\subsubsection{Fonction relationnelle}

Nous appliquons l'équation (1), dans notre cas pratique, ainsi nous aurons les informations suivantes. Soit $G_{j}$ un groupe de la classe, $(1 \leq j \leq 6)$, l'application: $\varphi_{/ G_{j}}: G_{j} \rightarrow \mathbb{N}$ telle que,

$m_{i, j} \rightarrow \varphi_{/ G_{j}}\left(m_{i, j}\right)=n_{i, j}$, où $n_{i, j}=\sum_{\substack{k=1 \\ k \neq i}}^{5} r_{i, k}, r_{i, k}$ est le coefficient qui est déterminé par la quantification du degré d'amitié du membre $m_{i, j}$ avec le membre $m_{i, k}$, en adoptant, voir Tableau 1, l'échelle de Likert. Le Tableau 8, nous indique l'intensité du relationnel de chaque membre de la classe dans son groupe. 
Tableau 8. Intensité de la relationnelle de chaque membre de la classe.

\begin{tabular}{|c|c|c|c|c|c|}
\hline $\boldsymbol{G}_{\mathbf{1}}$ & $\boldsymbol{\varphi}\left(\boldsymbol{G}_{\mathbf{1}}\right)$ & $\boldsymbol{G}_{\mathbf{2}}$ & $\boldsymbol{\varphi}\left(\boldsymbol{G}_{\mathbf{2}}\right)$ & $\boldsymbol{G}_{\mathbf{3}}$ & $\boldsymbol{\varphi}\left(\boldsymbol{G}_{\mathbf{3}}\right)$ \\
\hline$m_{1,1}$ & 16 & $m_{1,2}$ & 8 & $m_{1,3}$ & 14 \\
\hline$m_{2,1}$ & 13 & $m_{2,2}$ & 11 & $m_{2,3}$ & 16 \\
\hline$m_{3,1}$ & 11 & $m_{3,2}$ & 10 & $m_{3,3}$ & 16 \\
\hline$m_{4,1}$ & 16 & $m_{4,2}$ & 12 & $m_{4,3}$ & 14 \\
\hline$m_{5,1}$ & 14 & $m_{5,2}$ & 11 & $m_{5,3}$ & 14 \\
\hline $\boldsymbol{G}_{\mathbf{4}}$ & $\boldsymbol{\varphi}\left(\boldsymbol{G}_{\mathbf{4}}\right)$ & $\boldsymbol{G}_{\mathbf{5}}$ & $\boldsymbol{\varphi}\left(\boldsymbol{G}_{\mathbf{5}}\right)$ & $\boldsymbol{G}_{\mathbf{6}}$ & $\boldsymbol{\varphi}\left(\boldsymbol{G}_{\mathbf{6}}\right)$ \\
\hline$m_{1,4}$ & 13 & $m_{1,5}$ & 13 & $m_{1,6}$ & 13 \\
\hline$m_{2,4}$ & 13 & $m_{2,5}$ & 14 & $m_{2,6}$ & 19 \\
\hline$m_{3,4}$ & 15 & $m_{3,5}$ & 14 & $m_{3,6}$ & 16 \\
\hline$m_{4,4}$ & 11 & $m_{4,5}$ & 16 & $m_{4,6}$ & 17 \\
\hline$m_{5,4}$ & 13 & $m_{5,5}$ & 17 & $m_{5,6}$ & 16 \\
\hline
\end{tabular}

Ainsi, nous obtenons les vecteurs suivants :

$$
\begin{aligned}
& \varphi\left(G_{1}\right)=(16,13,11,16,14) \\
& \varphi\left(G_{2}\right)=(8,11,10,12,11) \\
& \varphi\left(G_{3}\right)=(14,16,16,14,14) \\
& \varphi\left(G_{4}\right)=(13,13,15,11,13) \\
& \varphi\left(G_{5}\right)=(13,14,14,16,17) \\
& \varphi\left(G_{6}\right)=(13,19,16,17,16)
\end{aligned}
$$

Nous concluons à partir des $M_{j}$ l'environnement d'amitié qui règne au sein de chaque groupe $G_{j}$, voir Tableau 9 .

Tableau 9. Moyenne et appréciation de l'environnement d'amitié de chaque groupe.

\begin{tabular}{|c|c|c|}
\hline Groupe & Moyenne d'amitié & Appréciation de l'environnement d'amitié \\
\hline$G_{1}$ & 14 & Bonne \\
\hline$G_{2}$ & 10.4 & Juste moyenne \\
\hline$G_{3}$ & 14.8 & Bonne \\
\hline$G_{4}$ & 13 & Assez bonne \\
\hline$G_{5}$ & 14.8 & Bonne \\
\hline$G_{6}$ & 16.2 & Très bonne \\
\hline
\end{tabular}

Nous avons $M=\frac{1}{6} \sum_{j=1}^{6} M_{j}=13.86$, nous indique que le climat relationnel qui règne au sein de la classe est bonne.

\subsubsection{Fonction de satisfaction}

Maintenant, nous appliquons l'équation (9), dans notre cas pratique, ainsi nous aurons les informations suivantes. Soit $G_{j}$ un groupe de la classe, $(1 \leq j \leq 6)$, nous considérons l'application,

$\theta_{/ G_{j}}: G_{j} \rightarrow \mathbb{N}$ telle que $m_{i} \rightarrow \theta_{/ G_{j}}\left(m_{i}\right)=s_{i}$, où, $s_{\mathrm{i}}$ est le coefficient qui est déterminé par la quantification du degré de satisfaction du membre $\mathrm{m}_{\mathrm{i}}$ dans son groupe, voir Tableau 4. Et par suite, nous aurons la fonction globale de satisfaction sur toute la classe notée $\theta$ définie de la façon suivante : 
Pour tout élève $E$ de la classe $C$, il existe i entre 1 et $r$, il existe un unique j entre 1 et $q$ tels que $E=m_{i, j}$ est dans $G_{j}, \theta(E)=\theta\left(m_{i, j}\right)=\theta_{\mid G j}\left(m_{i, j}\right)$.

Le Tableau 10 nous indique l'intensité de satisfaction de chaque membre de la classe dans son groupe.

Tableau 10. Intensité de la satisfaction de chaque membre de la classe.

\begin{tabular}{|c|c|c|c|c|c|}
\hline $\boldsymbol{G}_{\mathbf{1}}$ & $\boldsymbol{\theta}\left(\boldsymbol{G}_{\mathbf{1}}\right)$ & $\boldsymbol{G}_{\mathbf{2}}$ & $\boldsymbol{\theta}\left(\boldsymbol{G}_{\mathbf{2}}\right)$ & $\boldsymbol{G}_{\mathbf{3}}$ & $\boldsymbol{\theta}\left(\boldsymbol{G}_{\mathbf{3}}\right)$ \\
\hline$m_{1,1}$ & 4 & $m_{1,2}$ & 2 & $m_{1,3}$ & 3 \\
\hline$m_{2,1}$ & 3 & $m_{2,2}$ & 3 & $m_{2,3}$ & 4 \\
\hline$m_{3,1}$ & 2 & $m_{3,2}$ & 3 & $m_{3,3}$ & 5 \\
\hline$m_{4,1}$ & 5 & $m_{4,2}$ & 4 & $m_{4,3}$ & 4 \\
\hline$m_{5,1}$ & 4 & $m_{5,2}$ & 2 & $m_{5,3}$ & 4 \\
\hline $\boldsymbol{G}_{\mathbf{4}}$ & $\boldsymbol{\theta}\left(\boldsymbol{G}_{\mathbf{4}}\right)$ & $\boldsymbol{G}_{\mathbf{5}}$ & $\boldsymbol{\theta}\left(\boldsymbol{G}_{\mathbf{5}}\right)$ & $\boldsymbol{G}_{\mathbf{6}}$ & $\boldsymbol{\theta}\left(\boldsymbol{G}_{\mathbf{6}}\right)$ \\
\hline$m_{1,4}$ & 3 & $m_{1,5}$ & 3 & $m_{1,6}$ & 4 \\
\hline$m_{2,4}$ & 4 & $m_{2,5}$ & 3 & $m_{2,6}$ & 5 \\
\hline$m_{3,4}$ & 4 & $m_{3,5}$ & 3 & $m_{3,6}$ & 4 \\
\hline$m_{4,4}$ & 2 & $m_{4,5}$ & 4 & $m_{4,6}$ & 5 \\
\hline$m_{5,4}$ & 3 & $m_{5,5}$ & 5 & $m_{5,6}$ & 5 \\
\hline
\end{tabular}

Ainsi, nous obtenons les vecteurs suivants :

$$
\begin{aligned}
& \theta\left(G_{1}\right)=(4,3,2,5,4) ; \\
& \theta\left(G_{2}\right)=(2,3,3,4,2) ; \\
& \theta\left(G_{3}\right)=(3,4,5,4,4) ; \\
& \theta\left(G_{4}\right)=(3,4,4,2,3) ; \\
& \theta\left(G_{5}\right)=(3,3,3,4,5) ; \\
& \theta\left(G_{6}\right)=(4,5,4,5,5),
\end{aligned}
$$

Nous pouvons conclure à partir des $N_{j}$ l'environnement de satisfaction qui règne au sein de chaque groupe $G_{j}$, voit Tableau 11.

Tableau 11. Moyenne et l'appréciation de satisfaction de chaque groupe.

\begin{tabular}{|c|c|c|}
\hline Groupe & Moyenne de la satisfaction & Appréciation de l'environnement de satisfaction \\
\hline$G_{1}$ & 3.6 & Bonne \\
\hline$G_{2}$ & 2.8 & Assez bonne \\
\hline$G_{3}$ & 4 & Bonne \\
\hline$G_{4}$ & 3.2 & Assez bonne \\
\hline$G_{5}$ & 3.6 & Bonne \\
\hline$G_{6}$ & 4.6 & Très bonne \\
\hline
\end{tabular}

Nous avons $R=\frac{1}{6} \sum_{j=1}^{6} N_{j}=3.63$, cela nous indique que l'environnement de satisfaction qui règne au sein de la classe est bonne.

\subsubsection{Etude de l'impact du relationnelle sur la satisfaction des apprenants}

Nous mettons en évidence, voir Tableau 12, l'impact de l'environnement relationnel sur la satisfaction des membres de chaque groupe. En comparant les composantes correspondantes des deux vecteurs $\varphi\left(G_{j}\right)$ et $\theta\left(G_{j}\right)$, nous déterminons en valeur la corrélation entre le relationnel et le rendement des apprenants dans leur groupe. 
Tableau 12. Corrélation entre le relationnel et la satisfaction de chaque groupe.

\begin{tabular}{|c|c|c|c|c|c|c|c|c|c|}
\hline$\varphi\left(G_{1}\right)$ & $\boldsymbol{\theta}\left(\boldsymbol{G}_{1}\right)$ & $\varphi\left(G_{2}\right)$ & $\boldsymbol{\theta}\left(\boldsymbol{G}_{2}\right)$ & $\varphi\left(G_{3}\right)$ & $\boldsymbol{\theta}\left(\boldsymbol{G}_{3}\right)$ & $\varphi\left(G_{4}\right)$ & $\boldsymbol{\theta}\left(\boldsymbol{G}_{4}\right)$ & $\varphi\left(G_{5}\right)$ & $\theta\left(G_{5}\right)$ \\
\hline 16 & 4 & 8 & 2 & 14 & 3 & 13 & 3 & 13 & 3 \\
\hline 13 & 3 & 11 & 3 & 16 & 4 & 13 & 4 & 14 & 3 \\
\hline 11 & 2 & 10 & 3 & 16 & 5 & 15 & 4 & 14 & 3 \\
\hline 16 & 5 & 12 & 4 & 14 & 4 & 11 & 2 & 16 & 4 \\
\hline 14 & 4 & 11 & 2 & 14 & 4 & 13 & 3 & 17 & 5 \\
\hline$\varphi\left(G_{6}\right)$ & $\theta\left(G_{6}\right)$ & & & & & & & & \\
\hline 13 & 4 & & & & & & & & \\
\hline 19 & 5 & & & & & & & & \\
\hline 16 & 4 & & & & & & & & \\
\hline 17 & 5 & & & & & & & & \\
\hline 16 & 5 & & & & & & & & \\
\hline
\end{tabular}

Nous avons ainsi, les deux vecteurs qui sont définis dans l'équation (17),

$\boldsymbol{M} \boldsymbol{M}=(14,10.4,14.8,13,14.8,16.2) \quad$ et $\boldsymbol{R} \boldsymbol{R}=(3.6,2.8,4,3.2,3.6,4.6)$.

Pour mettre en évidence l'importance de la relationnelle sur le rendement de tous les membres de la classe, nous déterminons la corrélation entre les deux vecteurs $\boldsymbol{M} \boldsymbol{M}$ et $\boldsymbol{R} \boldsymbol{R}$.

\subsection{Interprétations des résultats}

Nous présentons dans ce paragraphe, la corrélation qui peut exister entre le climat relationnel qui règne au sein de chaque groupe et la progression dans l'acquisition des différents savoirs par les membres de ce groupe. En effet, Fig.1 montre l'effet de l'environnement relationnel et psychologique des apprenants sur leurs motivations à acquérir des nouvelles compétences. Les deux aspects relationnel et satisfaction ont la même allure de croissance, ce qui montre que la corrélation entre eux est très intéressante.
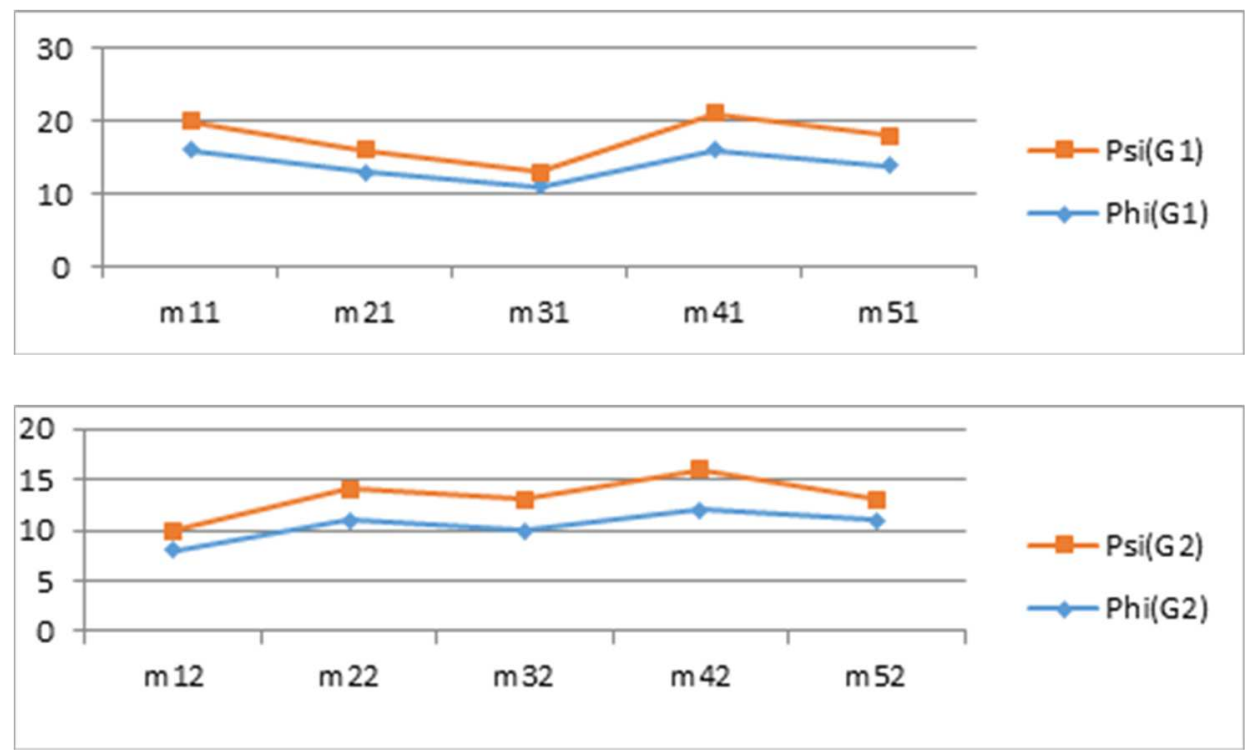

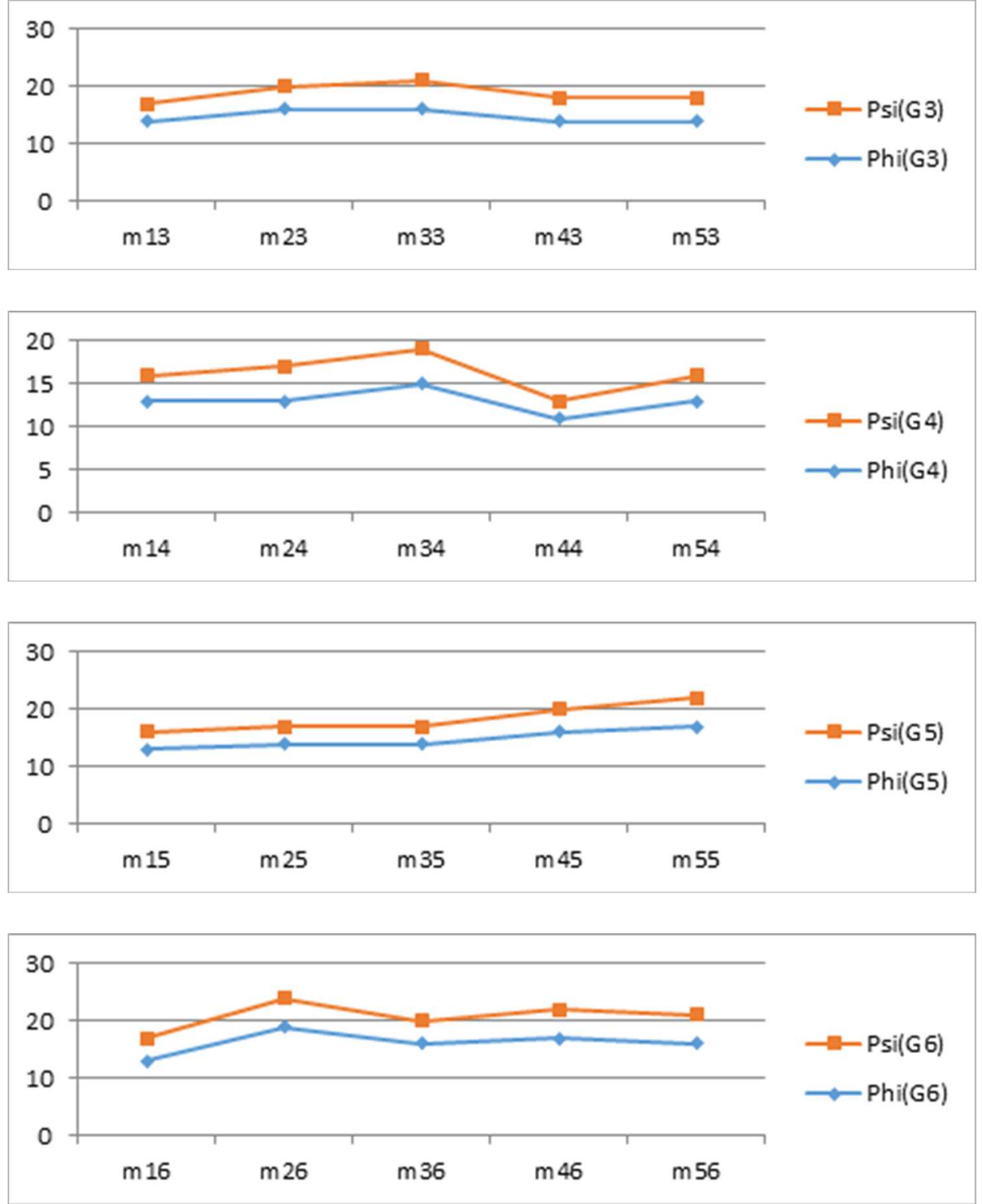

Fig. 1. Sens de variations des deux fonctions relationnelle et de satisfaction de chaque groupe.

Nous remarquons (Figure 1) que l'aspect relationnel qui règne au sein de chaque groupe à un impact positif dans le même sens de monotonie sur la satisfaction des apprenants dans ce groupe.

Dans une vision globale, l'aspect relationnel qui règne au sein de la classe, voir Fig. 2, à un impact positif dans le même sens de croissance sur la satisfaction des apprenants dans toute la classe. L'approche pédagogique adaptée par l'enseignant est plus efficace lorsque les apprenants interagissent dans leur environnement avec motivations. 


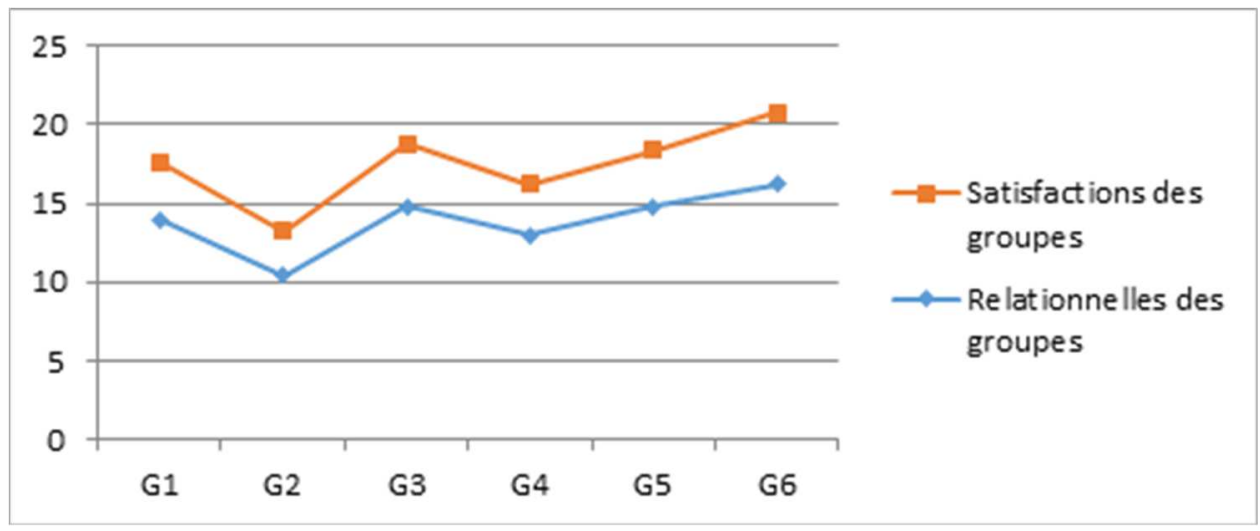

Fig. 2. Sens de variations des deux fonctions relationnelle et de satisfaction de la classe.

\section{Conclusion}

Dans cette recherche, nous avons montré l'importance de s'intéresser à l'aspect relationnel dans toute partition de la classe en adoptant la pédagogie différenciée. Son importance est due à la satisfaction des apprenants au sein de leurs groupes et aussi au sein de leur groupeclasse pour un meilleur rendement. Le caractère psychologique est très profondément présent dans l'opération d'apprentissage, ainsi le soutien nécessaire entre camarades-amis est fortement existant et la difficulté rencontrée est facilement surmontée par le groupe tout entier. En adoptant une méthode pédagogique active en animant la totalité de la classe sur les performances locales se percutent sur le niveau de la classe entière. Pour les enseignants qui utilisent la pédagogie différenciée doivent s'intéresser plus à l'effet psychologique de l'apprenant dans son groupe pour lui permettre une auto-évaluation motivante. L'étude que nous avons faite sur cette classe montre que lorsque nous préparons l'environnement pédagogique convenable aux apprenants, nous augmentons le pourcentage de réussite de notre opération d'apprentissage. A cet effet, l'enseignant doit s'intéresser plus à l'aspect relationnel entre tous les membres de la classe en adoptant des activités parallèles pour permettre aux apprenants de passer à une étape de camarades-amis. L'enseignant doit être conscient de l'impact du climat pédagogique qui se trouve dans sa classe sur l'efficacité de sa méthode pour interagir avec ses élèves.

\section{RÉFÉRENCES}

1. R. W. Burns. Postulats de Burns, Essor des didactiques et des apprentissages scolaires, JP ASTOLFI, 1995, (1972).

2. E. Touchard. La différenciation pédagogique comment faire ? Atelier encadré cycle 2 et 3. Circonscription Grenoble 4, (2011).

3. S. Herreman, P. Ghrenassia, C. Roye. Objectif CRPE Éducation et système éducatif. Hachette Éducation, (2017).

4. Y. Volpe. Individualisation, personnalisation, pédagogie différenciée ou différenciation : quelques clarifications conceptuelles. Educateur, no. 2, pp. 32-34, (2017).

5. E. Touchard. La différenciation pédagogique en mathématiques, Stage Mathématiques Cycles 2 et 3, 22 Mars 2012, CPC Grenoble 4, (2012). http://www.ac-grenoble.fr /mathssciences/IMG/pdf_Differencier_en_mathematiques_E_Touchard.pdf. 
6. J-P Roux. Socio-constructivisme et apprentissages scolaires. En ligne : dcalin.fr/fichiers/ jproux.doc, (2001).

7. L. S. Vygotsky. Thinking and speech. In R.W. Rieber \& A.S. Carton (Eds.), The collected works of L.S. Vygotsky, Volume 1: Problems of general psychology, pp. 39285, (1987).

8. F. Clerc. Changements dans la professionnalité enseignante, XYZep, 23, pp. 3-6, (2006).

9. S. Connac. La personnalisation des apprentissages. Agir face à l'hétérogénéité, à l'école et au collège. Paris: ESF Éditeurs, (2012).

10. C. Tyng, H. Amin, M. Saad, A. Malik, The Influences of Emotion on Learning and Memory. Front. Psychol. 8:1454, (2017). doi: 10.3389/fpsyg.2017.01454.

11. S. A. McLeod. Lev Vygotsky, (2018). Retrieved from https://www.simplypsychology.org/vygotsky.html

12. B. Bernstein-Yamashiro, G. G. Noam. Special Issue : Teacher-student relationships: Toward personalized education. New Directions for Youth Development, 137, (2013).

13. D. L. Roorda, H. M. Y. Koomen, J. L. Spilt, F. J. Oort. The influence of affective teacher-student relationships on students' school engagement and achievement: A meta-analytic approach. Review of Educational Research, 81, pp. 493-529, (2011).

14. G. Hagenauer and S. E. Volet. Teacher-student relationship at university: an important yet under-researched field. Oxford Review of Education, 40(3), pp. 370-388, (2014).

R. Croxton. The role of interactivity in student satisfaction and persistence. Journal of Online Learning \& Teaching, 10(2), pp. 314-324, (2014). 